\title{
PATHOLOGY OF ZOO ANIMALS AT THE UNIVERSITY OF IBADAN ZOOLOGICAL GARDEN
}

\author{
ANGA, T.J. AND AKPAVIE S. O. \\ Department of Veterinary Pathology \\ University of Ibadan \\ Ibadan, Nigeria
}

\section{SUMMARY}

A retrospective examination of post mortem records (1969 - 1990) revealed that 132 cases of zoo animals were presented for post-mortem. The common conditions diagnosed include gastroenteritis, respiratory disease and malnutrition/starvation which accounted for $70 \%$ of cases encountered. Other conditions were tumours, chemical poisoning, anthrax and actinomycosis.

KEY WORDS: Pathology, Zoo Animals, Zoological Garden.

\section{INRODUCTION}

Keymer (1974) reported that information on the pathology of zoo animals was very scanty. Until recently, little work has been done to find out the types of parasites found in these animals (Crockett, 1983). Work done on wildlife diseases has been initiated because the problem under investigation was of zoonotic importance or is related to the health of domestic animals (Jones, 1982).

A few conditions, anthrax, verminous penumonia and neoplasia have been reported at the Ibadan zoological Gardens (Idowu et al_1975; Ikede et al., 1976 and Akinyemi and Ikede, 1982). It is also known that although most primates in the zoo are mainly vegetarians, they require a certain amount of animal protein. Therefore. their diet should vary, and growing and breeding animals should be supplemented with vitamins and minerals (New, 1966).

In this report, we highlight the various conditions diagnosed in zoo/wild animals using both gross and histopathological studies.

\section{MATERIALS AND METHODS}

\section{Animals}

Animals used for this study were those kept at the University of Ibadan Zuological Garden.

Records of postmortem performed on zoo/wild animals kept at the department of veterinary pathology, University of Ibadan between 1969 and 1990 were also used. 


\section{NIGERIAN VETERINARY JOURNAL}

\section{Housing}

The animals at the zoo are kept in various types of enclosures and the carnivorous ones are fed on slaughtered small ruminants and cattle meat, the herbivores on grasses and shrubs while the omnivorous animals feed on fruits.

\section{Pathology}

At post mortem, tissues were fixed in 10\% buffered formalin, processed routinely for histopathology and stained with haematoxylin and eosin ( $\mathrm{H}$ and $\mathrm{E}$.). Special staining was done using Martius Scarlet Blue (MSB), Periodic Acid Schiff (PAS.)

\section{RESULTS}

The results of the pathological conditions observed in zoo animals at post mortem are shown in Tables I - VI.

Many of the animals examined were observed to have died of pneumonia, gastroenteritis, malnutrition and septicaemic conditions, although few had neoplastic conditions, while others died of poisoning or toxaemia. In rare cases trauma due to bullet wounds and heart water/hepatitis were diagnosed.

\section{DISCUSSION}

During this study period (1969 - 1990) various pathological conditions were diagnosed in the animals kept at the University of Ibadan Zoological Garden: Gastroenteritis was the most common lesion observed. The common occurrence of this lesion was associated with intestinal parasites and the interplay between factors such as change in diet and unsuitable diets. One of the most important effects of this lesion which is malabsorption has been previously reported (Keymer, 1974).

The next commonly encountered lesion in this study was pneumonia. The occurrence of this condition was, ittributed to various infectious agents (parasitic, bacterial, mycotic and viral agents). Petrak (1982) reiterated that inclement weather can play a role in the development of pneumonia as been reported for non-caged domestic animals. Malnutrition and starvation were also seen in several animals. These conditions were attributed to poor management and feeding as there was no record of supplementation of the diets of the animals in the zoo with any vitamins or minerals. Septicaemic of supple conditions like anthrax were observed. In the case of anthrax 2 genets died and infection was associated with beef obtained from the abattoir that was fed to the animals (Ikede et al. 1976).

Trauma caused by bullets was not common, but two cases, one in an antelope and another in a mangabay were diagnosed. It is noteworthy that animals that cause injuries to others are usually eliminated by shooting. Also, not common was heart water, which was diagnosed in one deer once.

Although tumours were infrequent, two cases of fibroma were observed in reptiles (ldowu et al., 1975).

Gout was a frequent finding in birds in the collection. The condition is usually 
associated with excessive protein consumption or insufficient intake of water (Petrak, 1982).

\section{ACKNOWLEDGEMENTS}

The authors are grateful to members of staff of the Department of Veterinary Pathology who took part in the examination of carcasses.

\section{REFERENCES}

AKINYEMI, J. O. and IKEDE, B. O. (1982): Verminous pneumonia in a Gabon Viper,Bitis gabonica. Zo. Garten. 52:78 - 80 .

CROCKETT, E. C. (1983): A preliminary survey of some helminth parasites of game animals in Kainji Lake National Park, Borgu Sector. M. Sc. Dissertation, University of Ibadan.

GORDON, H. MCL and WHITELOCK, H. V. (1939): A new technique for counting nematode eggs in sheep faeces. J. Council Sc. Ind. Res. Aust. 12 - 50.
IDOWU, A. L., GOLDING, R. R., IKEDE, B. O. and HILL, D. H. (1975): Oral fibroma in captive python. J. Wldl. Dis. 11:201 - 204.

IKEDE, B. O., FALADE, $S$ and GOLDING R. R. (1976): Anthrax in captive carnivores in Ibadan, Nigeria. J. Wlal Dis. 12:130 - 132.

JONES, D. (1982): The Veterinary Surgeon and wildlife conservation. Vet. Rec :111: 427 - 431.

KEYMER, I. F. (1974): Report of the Pathologist In: Scientific Report, 1971 - 1973. The Zoological Society of London. J. Zool. Lon. 173:59.

NEW, A. E. (1966): Primate Management and Therapeutics. Small Anim. Clin 61:448.

PETRAK, M. L. (1982): Diseases of Cage and Aviary birds. $2^{\text {nd }}$ Ed. Lea and Febiger, Philadelphia. 
TABLE I: Pathological conditions in ruminants

\begin{tabular}{|c|c|c|c|c|}
\hline \multirow[t]{2}{*}{ Animals } & No. of Cases & \multicolumn{2}{|c|}{ Diagnosis } & \multirow{2}{*}{$\frac{\text { Number (\%) }}{1(4.76)}$} \\
\hline & & (i) & Necrotic abomasitis & \\
\hline & & (ii) & Pneumonia & $2(9.52)$ \\
\hline & & (iii) & Shock & $2(9.52)$ \\
\hline Maxwell & & (iv) & Purulent adenitis (Actinomycosis) & $1(4.76)$ \\
\hline \multirow[t]{7}{*}{ Diukers } & 21 & (v) & Fibrinous pericarditis/Pleurisy & $3(14.28)$ \\
\hline & & (vi) & Chemical poisoning & $4(19.0)$ \\
\hline & & (vii) & Starvation & $1(4.76)$ \\
\hline & & (viii) & Gangrenous Stomatitis & $1(4.76)$ \\
\hline & & (ix) & Pulmonary Congestion & $1(4.76)$ \\
\hline & & $(x)$ & None & $6(28.52)$ \\
\hline & & (i) & Pasteurellosis/Enterotoxaemia & $1(50)$ \\
\hline \multirow[t]{2}{*}{ Antelopes } & 2 & (ii) & Gun shot wound & $1(50)$ \\
\hline & & (i) & Anaemia/Haemorrhagic enteritis & $1(33.3)$ \\
\hline \multirow[t]{3}{*}{ Bush bucks } & 3 & (ii) & None & $2(66.67)$ \\
\hline & & (i) & Gastroenteritis & $3(60.0)$ \\
\hline & & (ii) & Toxaemia & $1(20.0)$ \\
\hline \multirow[t]{3}{*}{ Elephants } & 5 & (iii) & Intravascular haemolysis & $1(20.0)$ \\
\hline & & (iv) & Helminthiasis (strongylosis) & $1(20.0)$ \\
\hline & & (i) & Heart water/Hepatitis & $1(33.33)$ \\
\hline \multirow[t]{2}{*}{ Deers } & 3 & (ii) & None & $2(66.67)$ \\
\hline & & (i) & Haemorrhagic enteritis/Pulmonary & $1(50.0)$ \\
\hline Hippopotamus & 2 & & Congestion & $1(50.0)$ \\
\hline i: & & (ii) & None & \\
\hline
\end{tabular}

TABLE II: Pathological conditions in carnivores

\begin{tabular}{|c|c|c|c|c|}
\hline Animals & $\begin{array}{l}\text { No. of Cases } \\
\text { Examined }\end{array}$ & & Diagnosis & $\begin{array}{c}\text { Number } \\
(\%)\end{array}$ \\
\hline & & (i) & Salmonella enteritis & $1(50.00)$ \\
\hline Civet cats & 2 & (ii) & None & $1(50.00)$ \\
\hline \multirow[t]{2}{*}{ Australian skunks } & 1 & (i) & Cannibalism & $1(100.00)$ \\
\hline & & (i) & Cannibalism & $1(20.00)$ \\
\hline Mangoose & & (ii) & Ulcerative haemorrhagic enteritis & $1(20.00)$ \\
\hline \multirow[t]{2}{*}{ Lemurs } & 5 & (iii) & Skin tumour & $1(2000)$ \\
\hline & & (iv) & None & $1(20.00)$ \\
\hline Caracals & 1 & (i) & Septicaemia & $1(100.00)$ \\
\hline \multirow[t]{2}{*}{ Genets } & 2 & (i) & Anthrax & $2(100.00)$ \\
\hline & & (i) & Malnutrition & $1(50.0)$ \\
\hline Hyaenas & 2 & (ii) & Euthanasia & $1(50.0)$ \\
\hline \multirow[t]{2}{*}{ Lions } & 2 & (i) & Malnutrition & $1(50.00)$ \\
\hline & & (ii) & Granulomatous bronchopneumonia & $1(50.00)$ \\
\hline Red River & & (i) & Interstitial pneumonia/ acute balanoposthitis & $1(50.00)$ \\
\hline Hog & 2 & (ii) & Haemorrhagic diathesis. & $1(50.00)$ \\
\hline
\end{tabular}




\section{TABLE III: Pathological conditions in primates}

\begin{tabular}{|c|c|c|c|c|}
\hline Animals & $\begin{array}{l}\text { No. of Cases } \\
\text { Examined }\end{array}$ & & Diagnosis & $\begin{array}{l}\text { Number } \\
(\%)\end{array}$ \\
\hline \multirow{11}{*}{ Monkeys } & \multirow{11}{*}{18} & (i) & Helminthosis (Trichuriasis) & $5(26.32)$ \\
\hline & & (ii) & Metatarsal fracture with exostosis & $1(5.26)$ \\
\hline & & (iii) & Encephalitis & $1(5.26)$ \\
\hline & & (iv) & Ulcerative oesophagitis & $1(5.26)$ \\
\hline & & $(v)$ & Fibrinous pleuritis & $1(5.26)$ \\
\hline & & (vi) & Bacterial septicaemia & $2(10.52)$ \\
\hline & & (vii) & Ruptured diaphragm & $\{(5.26)$ \\
\hline & & (viii) & Haemorrhagic tracheobronchitis/fatty liver & $1(5.26)$ \\
\hline & & (ix) & Lung worms/head trauma & $1(5.26)$ \\
\hline & & $(\mathrm{x})$ & None & $4(21.05)$ \\
\hline & & (i) & Haemorrhagic tracheobronchitis & $1(50.00)$ \\
\hline Mangabays & 2 & (ii) & Gun shot wound & $1(50.00)$ \\
\hline \multirow[t]{3}{*}{ Baboon } & \multirow[t]{3}{*}{1} & (i) $\mathrm{Ha}$ & morrohagic bronchopneumonia & $1(100.00)$ \\
\hline & & (i) & Oesteodystrophia fibrosa. & $1(20.00)$ \\
\hline & & (ii) & Toxaemia/nutritional deficiency & $1(20.00)$ \\
\hline \multirow[t]{4}{*}{ Pottos } & \multirow[t]{4}{*}{5} & (iii) & Simian bone disease & $1(20.00)$ \\
\hline & & (iv) & Haemorrhagic enterocolitis & $1(20.00)$ \\
\hline & & $(v)$ & None & $1(20.00)$ \\
\hline & & (i) & Patent foramen ovale & $1(50.00)$ \\
\hline \multirow[t]{2}{*}{ Chimpanzees } & \multirow[t]{2}{*}{2} & (ii) & None & $1(50.00)$ \\
\hline & & (i) & Cerebral haemorrhage (fracture of the skull) & $1(50.00)$ \\
\hline \multirow[t]{3}{*}{ Simians } & \multirow[t]{3}{*}{2} & (ii) & Haemorrhagic tracheobronchitis/fatty liver & $1(50.00)$ \\
\hline & & (i) & Starvation/pulmonary congestion and oedema & $1(25.00)$ \\
\hline & & (ii) & Haemorrhagic enteritis & $1(25.00)$ \\
\hline \multirow[t]{2}{*}{ Others } & 4 & (iii) & $\begin{array}{l}\text { Helminthiosis (Trichuriasis)/non-suppurative } \\
\text { encephalitis }\end{array}$ & $1(25.00)$ \\
\hline & & (iv) & None & $1(25.00)$ \\
\hline
\end{tabular}




\section{NIGERIAN VETERINARY JOURNAL}

TABLE: IV: Pathological conditions in reptiles

\begin{tabular}{|c|c|c|c|c|}
\hline Animals & $\begin{array}{l}\text { No. of Cases } \\
\text { Examined }\end{array}$ & & Diagnosis & $\begin{array}{c}\text { Number } \\
(\%)\end{array}$ \\
\hline \multirow{7}{*}{ Pythons } & \multirow[t]{7}{*}{ 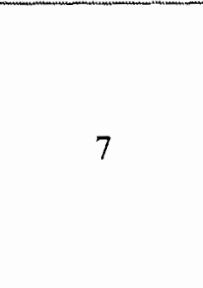 } & (i) & Granulomatous myositis & $1(16.67)$ \\
\hline & & (ii) & Fibroma & \\
\hline & & (iii) & Cornifying epithelioma & $\mathrm{I}(16.67)$ \\
\hline & & (iv) & Ulcerative enteritis /Pulmonary & \\
\hline & & & congestion & $1(16.67)$ \\
\hline & & $(v)$ & Helminthiasis & $1(16.67)$ \\
\hline & & (vi) & Rectal hair ball & $1(16.67)$ \\
\hline \multirow[t]{2}{*}{ Viper } & \multirow[t]{2}{*}{1} & (i) & Verminous pneumonia & \multirow[t]{2}{*}{$1(100.00)$} \\
\hline & & (i) & Instestinal torsion with peritonitis & \\
\hline \multirow[t]{2}{*}{ Tortoise } & \multirow[t]{2}{*}{2} & & and Toxaemia & $1(50.00)$ \\
\hline & & (ii) & Necrotizing enteritis & $1(50.00)$ \\
\hline Boa & 2 & (i) & Hepatoma/pneumonia & $1(50.00)$ \\
\hline Constrictors & & (ii) & Hepatitis & $1(50.00)$ \\
\hline \multirow[t]{2}{*}{ Rattle Snake } & 1 & (i) & Purulent Peumonia/catarthal entreritis & $1(100.00)$ \\
\hline & & (i) & Gangrenous dermatitis & $1(50.00)$ \\
\hline Others & 2 & (ii) & Ulcerated fibroma & $1(50.00)$ \\
\hline
\end{tabular}

TABLE V: Pathological conditions in avian

\begin{tabular}{|c|c|c|c|c|}
\hline Animals & $\begin{array}{l}\text { No. of Cases } \\
\text { Examined }\end{array}$ & \multicolumn{2}{|r|}{ Diagnosis } & $\begin{array}{c}\text { Number } \\
(\%)\end{array}$ \\
\hline & & (i) & Haemorrhagic gastroenteritis & $2(33.34)$ \\
\hline & & (ii) & Candidiasis & $1(16.67)$ \\
\hline \multirow[t]{2}{*}{ Parrots } & 6 & (iii) & Asphyxation & $1(16.670)$ \\
\hline & & (iv) & None & $2(33.34)$ \\
\hline Peacocks & 2 & (i) & Haemorrhagic enteritis & $2(100.00)$ \\
\hline \multirow[t]{2}{*}{ Bush Fowls } & 2 & (i) & Parasitic enteritis & $2(100.00)$ \\
\hline & & (i) & Haemorrhagic gastroenteritis & $2(100)$ \\
\hline \multirow[t]{2}{*}{ Ostriches } & 2 & (ii) & Mycotic pneumonia (Aspergi/lus fumiganus) & $1(50.00)$ \\
\hline & & (i) & Gout & $1(33.40)$ \\
\hline Flamingoes & 3 & (ii) & None & $22(66.67)$ \\
\hline Pelicans & 2 & (i) & Fracture of the skull (parietal bone) & $1(50.00)$ \\
\hline Pigeon & 1 & (i) & Autolysis & $1(100.00)$ \\
\hline Mynal bird & 1 & (i) $\mathrm{U}$ & ilateral suppurative ophthalmitis & $1(100.00)$ \\
\hline \multirow[t]{2}{*}{ Rock Hyrax } & 1 & (i) $\mathrm{A}$ & cute peritonitis & $1(100.00)$ \\
\hline & & (i) & Gout & $3(50.00)$ \\
\hline \multirow[t]{3}{*}{ Others } & 6 & (ii) & Organophosphorus poisoning & $1(16.33)$ \\
\hline & & (iii) & Air Sacculitis/ascariasis & $1(16.33)$ \\
\hline & & (iv) & Capillaria infection. & $1(16.33)$ \\
\hline
\end{tabular}


ANGA \& AKPAVIE: PATHOLOGY OF Z00 ANIMALS AT IBADAN ZOOLOGICAL GARDEN

TABLE VI: Pathological conditions in other animals

\begin{tabular}{lcccc}
\hline Animals & No. of Cases Examined & Diagnosis & Number $(\%)$ \\
Cotimudi & 2 & (i) & Viral Hepatitis & $1(50.00)$ \\
Sitatunga & 1 & (ii) & None & $1(50.00)$ \\
& & (i) & Asphyxation & $1(0.00)$ \\
Murines & 2 & (i) & Gastroenteritis & $1(50.00)$ \\
\hline
\end{tabular}

